P219 CHALLENGES WITH END-OF-LIFE CARE IN COVID PATIENTS REQUIRING NON-INVASIVE RESPIRATORY SUPPORT

RE Nixon, SB Naidu, J Fretwell, K Matsumoto, F Rashid, T Sutton, Z Thursz. Whipps Cross University Hospital, London, UK

\subsection{6/thorax-2021-BTSabstracts.328}

Introduction The COVID-19 pandemic has seen an unprecedented number of adults receiving non-invasive respiratory support (NIRS) with such patients having a high mortality rate.

Methods As part of better elucidating the challenges of end of life care delivery in the COVID era, we conducted an audit of our respiratory HDU ward at Whipps Cross Hospital focusing on a 19-week period between 17/09/2020-30/01/ 2021 and on patients who did not survive their admission. We excluded patients that were transferred to ITU.

Results Of a total of 309 patients receiving NIRS on our ward, 84 died during that time at a mean age of $77(95 \%$ CI 67-87) and median of 79 years. 63 patients received CPAP, 67 received HFNT and 42 were first started on HFNT and converted to CPAP. The average length of stay was 10 days (4-16). The mean day of symptoms on presentation to hospital was 11.5 days (1.7-21.3). Average duration of symptoms prior to admission to our ward was 19.7 (9.130.3) days.

One death was unexpected and followed a cardiac arrest. The most common indicator for a patient approaching end-oflife was hypoxia on NIRS, which was documented in 36 $(43 \%)$ patients, followed by terminal agitation in $27(32 \%)$ patients. The average time between recognising end-of-life and death was 1.4 days with a median of 2 days. 72 (86\%) patients were weaned off NIRS and those who continued did so due to a medical or patient decision. Despite the vast majority ( $82 \%$ of patients) being on syringe drivers with an opiate and benzodiazepine most patients had persistent terminal symptoms: 51 (74\%) had agitation and 38 (55\%) were persistently breathlessness. Interestingly, no patient opted to rest in the prone position.

Discussion This data primarily suggests the challenging nature of managing end-of-life care for COVID patients deteriorating on NIRS due to the high symptom load and the short time there is to achieve comfort for these individuals. Clinicians need to conduct frequent comfort reviews for such patients, consider subcutaneous infusions, as well as potentially an increase in medication doses, in conjunction with specialist palliative care input, in order to achieve comfort.

\section{P220 ASSESSING THE MULTI-DISCIPLINARY TEAM RESPONSE TO NIV WITHDRAWAL GUIDELINES IN PATIENTS WITH COVID-19}

A Birtles, K Gaffney, G Mullen, A Boland. Leeds Teaching Hospitals NHS Trust, Leeds, UK

\subsection{6/thorax-2021-BTSabstracts.329}

Introduction Withdrawal of NIV in COVID-19 patients at end of life presents several challenges. Patients are often more alert and have a higher symptom burden than in other end of life situations where NIV is withdrawn. The NIV withdrawal guideline, created by the centre, was updated to reflect the requirement for higher doses of anticipatory medications required for some patients in this cohort after learning from the first wave of COVID-19. The aim of this study was to review staff response to the guideline and its efficacy.

Method A questionnaire was sent to physician associates, nursing staff and doctors of all grades who have worked on the Respiratory Support Unit during the COVID-19 pandemic. This collected several types of data on staff perception of NIV withdrawal in COVID-19 patients.

Results The questionnaire generated 39 responses from the multidisciplinary team (MDT).

$97 \%$ of respondents found the withdrawal of NIV in COVID-19 challenging, and 74\% felt this was more difficult in patients with COVID-19 than with other pathologies. $87 \%$ were aware of the Trust guideline regarding NIV withdrawal and $82 \%$ used it in their practice. All respondents felt the guideline was useful. While the majority of healthcare workers felt that adequate symptom control was achieved, 20\% of respondents did not. This unease was further evidenced as $64 \%$ of respondents had issues or concerns regarding the use of anticipatory medications. The predominant concerns were that medication doses were started too low $(35 \%)$ or too late (46\%). $71 \%$ of respondents found discussions with families regarding commencing palliation challenging. All members of the multidisciplinary team found an MDT approach, including the involvement of Palliative Care colleagues, a useful source of support. The team was united in finding debriefs useful.

Conclusions Overall, this study identified that timing and dosage of anticipatory medications are a particular challenge in withdrawal of NIV in patients with COVID-19. There is scope for additional learning regarding symptom management during withdrawal of NIV. Maintaining a close relationship with the Palliative Care team provides benefit to patients, their families and staff. Further work will also focus on supporting staff in difficult conversations. 